\title{
ANOTHER CHARACTERIZATION OF BMO
}

\author{
R. R. COIFMAN ${ }^{1}$ AND R. ROCHBERG ${ }^{1}$
}

\begin{abstract}
The following characterization of functions of bounded mean oscillation (BMO) is proved. $f$ is in BMO if and only if

$$
f=\alpha \log g^{*}-\beta \log h^{*}+b
$$

where $g^{*},\left(h^{*}\right)$ is the Hardy-Littlewood maximal function of $g,(h)$, respectively, $b$ is bounded and $\|f\|_{\mathrm{BMO}}<c\left(\alpha+\beta+\|b\|_{\infty}\right)$.
\end{abstract}

I. Introduction. A real-valued locally integrable function $f(x)$ defined on $\mathbf{R}^{n}$ is said to be in BMO, the space of functions of bounded mean oscillation, if

$$
\sup _{Q} \frac{1}{|Q|} \int_{Q}\left|f(x)-m_{Q}(f)\right| d x<\infty
$$

where the supremum is over all cubes $Q$ in $\mathbf{R}^{n},|Q|$ denotes the volume of $Q$, and

$$
m_{Q}(f)=\frac{1}{|Q|} \int_{Q} f(x) d x
$$

The space of such functions modulo constant functions is a Banach space with the norm given by the left-hand side of (1).

If $\mu$ is a positive locally finite Borel measure defined on $\mathbf{R}^{n}$ then $\mu^{*}$, the maximal function of $\mu$, is defined by

$$
\mu^{*}(x)=\sup \{\mu(Q) /|Q| ; Q \text { is a cube containing } x\} .
$$

Let $L^{+}$denote the set of nonnegative locally integrable functions on $\mathbf{R}^{n}$. If $f$ is in $L^{+}$then $f^{*}$, the maximal function of $f$, is defined to be the maximal function of the measure $f(x) d x$. Our main result is the following.

THEOREM. There is a constant $c$ (which depends only on $n$ ) such that if $\alpha$ and $\beta$ are positive constants, $g$ and $h$ are in $L^{+}$with $g^{*}$ and $h^{*}$ finite a.e., and $b$ is any bounded function then the function

$$
f(x)=\alpha \log g^{*}(x)-\beta \log h^{*}(x)+b(x)
$$

is in $B M O$ and

$$
\|f\|_{B M O} \leqslant c\left(\alpha+\beta+\|b\|_{\infty}\right) .
$$

Conversely, if $f$ is any function in BMO then $f$ can be written in the form (2) with

$$
\alpha+\beta+\|b\|_{\infty}<c\|f\|_{B M O} .
$$

Received by the editors February 1, 1978.

AMS (MOS) subject classifications (1970). Primary 44A25.

${ }^{1}$ This research was supported in part by National Science Foundation Grants MCS75-02411 A02 and MCS76-05789, respectively. 
The proof that any function of the form (2) is in BMO is direct and is presented in $\$ 2$ along with some observations which may be of independent interest. Our proof that any BMO function can be written in the form (2) is a rather easy consequence of a deeper result of $\mathrm{L}$. Carleson characterizing BMO functions as the images of bounded functions under "adjoint maximal operators" [1]. This derivation is in $\$ 3$. The final section contains some notes and questions.

Many of these ideas were developed in conversations with Guido Weiss whose contributions we gratefully acknowledge.

II. Logarithms of weights and BLO. We will say that a locally integrable function $b$ has bounded lower oscillation ( $b$ is in BLO) if there is a constant $c$ such that for any cube $Q$

$$
m_{Q}(b)-\inf _{Q} b \leqslant c .
$$

If $b$ satisfies this condition then $b$ is also in BMO and $\|b\|_{\mathrm{BMO}} \leqslant 2 c$. To see this we use the fact that for any cube $Q$, the function $m_{Q}(b)-b(x)$ has mean zero on $Q$. Thus

$$
\begin{aligned}
\frac{1}{|Q|} \int_{Q}\left|m_{Q}(b)-b(x)\right| d x & =\frac{2}{|Q|} \int_{Q} \max \left(m_{Q}(b)-b(x), 0\right) d x \\
& \leqslant \frac{2}{|Q|} \int_{Q}\left(m_{Q}(b)-\inf _{Q} b\right) d x \leqslant 2 c .
\end{aligned}
$$

Functions in BLO are exactly logarithms of weights in Muckenhoupt's class $A_{1}$ [7]. A function $\omega(x)$ in $L^{+}$is said to be in class $A_{1}$, if there is a constant $c$ such that for all cubes $Q$

$$
m_{Q}(\omega) \leqslant \underset{Q}{\inf } \omega
$$

or equivalently, there is a constant $c$ such that

$$
\omega^{*}(x) \leqslant c \omega(x) \text { a.e. }
$$

LEMMA $1 . f$ is in BLO if and only if $e^{e f}$ is in $A_{1}$ for some positive $\varepsilon$.

Proof. If $f$ is in BLO then, by the theorem of John and Nirenberg [5], if $\varepsilon$ is sufficiently small then for some constant $c$ and for all cubes $Q$

$$
\frac{1}{|Q|} \int_{Q} \exp \left(\varepsilon\left|f-m_{Q}(f)\right|\right) d x \leqslant c .
$$

Dropping the absolute values and rearranging yields

$$
m_{Q}\left(e^{e f}\right) \leqslant c e^{m_{Q}(e f)} \text {. }
$$

An application of (3) now yields (4.1) and shows $e^{e f}$ is in $A_{1}$.

If $e^{e f}$ is in $A_{1}$ then taking logarithms in (4.1) and an appeal to Jensen's inequality shows $f$ is in BLO.

The following proposition establishes the relationship between $A_{1}$ weights and maximal functions. 
Proposition 2. If $\mu$ is a locally finite positive Borel measure with $\mu^{*}(x)<\infty$ a.e. and if $0<\delta<1$ then $\left(\mu^{*}\right)^{\delta}$ is in $A_{1}$.

Proof. Let $\mu$ and $\delta$ be given. Pick and fix a cube $Q$ and let $\bar{Q}$ be the double of $Q$. Let $\chi_{\bar{Q}}(x)$ be the characteristic function of $\bar{Q}$. Write $\mu=\mu_{1}+\mu_{2}$ where $\mu_{1}=\chi_{\bar{Q}} \mu$ and $\mu_{2}=\left(1-\chi_{\bar{Q}}\right) \mu$. Since $\mu^{* \delta} \leqslant c_{\delta}\left(\mu_{1}^{* \delta}+\mu_{2}^{* \delta}\right)$ it suffices to show

$$
m_{Q}\left(\mu_{i}^{* \delta}\right) \leqslant c \inf _{Q}\left(\mu^{* \delta}\right)
$$

for $i=1,2$ and some $c$ which does not depend on $Q$.

For $\mu_{1}$ we use Kolmogorov's inequality for the maximal function $([9$, p. 85]). This yields $\int_{\bar{Q}} \mu_{1}^{* \delta} \leqslant c_{\delta}|\bar{Q}|^{1-\delta}\left(\int_{\mathbf{R}^{n}} \mu_{1}\right)^{\delta}$. Hence $m_{Q}\left(\mu_{1}^{* \delta}\right) \leqslant c_{\delta} m_{Q}(\mu)^{\delta}$ which implies (5) for $i=1$.

For $\mu_{2}$ we use the obvious geometric estimate that if $x, y$ are in $Q$ then

$$
\mu_{2}^{*}(x) \leqslant c \mu_{2}^{*}(y)
$$

for some constant $c$ which depends only on the dimension. Hence

$$
\mu_{2}^{*}(x) \leqslant c \underset{Q}{c \inf _{Q}} \mu_{2}^{*} \leqslant c \underset{Q}{\inf } \mu^{*} .
$$

Raising both sides of this inequality to the power $\delta$, and then integrating over $Q$ establishes (5) for $i=2$ and completes the proof.

It should be noted that the constant $c$ in (5) does not depend on $\mu$.

This construction yields essentially all the elements of $A_{1}$ and in fact essentially all of $A_{1}$ is obtained using only measures of the form $f(x) d x$.

Corollary 3. (a) If $\omega(x)$ is in $A_{1}$ then there is an $F$ in $L^{+}, a \delta$ between 0 and 1 and a function $H$ which is bounded and bounded away from zero such that $\omega=F^{* \delta} H$.

(b) If $f$ is in $B L O$ then there is an $F$ in $L^{+}$, a positive number $\alpha$, and a bounded function $h$ such that

$$
f=\alpha \log F^{*}+h
$$

Proof. Suppose $\omega$ is in $A_{1}$. By the "reverse Hölder inequality" from the theory of weight [2], there is a positive $\varepsilon$ and a constant $c$ so that

$$
m_{Q}\left(\omega^{1+e}\right) \leqslant c m_{Q}(\omega)^{1+e}
$$

for all cubes $Q$. Combining this with (4.1) yields

$$
m_{Q}\left(\omega^{1+\varepsilon}\right)^{1 /(1+\varepsilon)} \leqslant c \inf _{Q} \omega
$$

hence

$$
\left(\left(\omega^{1+\varepsilon}\right)^{*}\right)^{1 /(1+\varepsilon)} \leqslant c \omega
$$

Certainly

$$
\left(\left(\omega^{1+e}\right)^{*}\right)^{1 /(1+\varepsilon)} \geqslant \omega .
$$

Hence setting $\delta=(1+\varepsilon)^{-1}, F=\omega^{1+\varepsilon}$ and $H=\left(F^{*}\right)^{-\delta} \omega$, gives the required decomposition. Part (b) of the corollary follows from part (a) by taking logarithms and appealing to Lemma 1. 
Note. In (6), $F$ can be chosen to be $e^{e f}$ for some small positive $\varepsilon$.

III. Proof of the theorem. The theorem follows from the results of the previous section and the fact which we now prove which is that any BMO function can be written as the difference of two BLO functions. This is a consequence of the following representation theorem for BMO functions due to L. Carleson [1]. ${ }^{2}$

THEOREM. Let $\varphi$ be a nonnegative Lipschitz function supported in the unit ball of $\mathbf{R}^{n}$ with $\int \varphi=1$. There are constants $c_{1}$ and $c_{2}$ such that if $\varepsilon(y)$ is any measurable function and $b_{1}$ and $b_{2}$ are bounded functions then

$$
f(x)=b_{1}(x)+\int \frac{1}{\varepsilon(y)^{n}} \varphi\left(\frac{x-y}{\varepsilon(y)}\right) b_{2}(y) d y
$$

is in $B M O$ and $\|f\|_{B M O} \leqslant c_{1}\left(\left\|b_{1}\right\|_{\infty}+\left\|b_{2}\right\|_{\infty}\right)$. Conversely, if $f$ is in BMO then $f$ can be written in the form (7) with functions $b_{1}$ and $b_{2}$ which satisfy $\left\|b_{1}\right\|_{\infty}+\left\|b_{2}\right\|_{\infty}<$ $c_{2}\|f\|$.

The decomposition we want follows from (7) by writing $b_{2}$ as the difference of two nonnegative functions. We must show that if $\varepsilon(y)$ and $\varphi$ are as above and $0<b(x)<k$ then

$$
g(x)=\int \frac{1}{\varepsilon(y)^{n}} \varphi\left(\frac{x-y}{\varepsilon(y)}\right) b(y) d y
$$

is in BLO and its BLO constant is dominated by some multiple of $k$. Let $Q$ be given and let $\bar{Q}$ be $Q$ scaled up by a factor of 5 . Write $b$ in (8) as $b=b_{1}+b_{2}$ with $b_{1}=b \chi_{\bar{Q}}\left(\chi_{\bar{Q}}\right.$ is the characteristic function of $\left.\bar{Q}\right)$ and $b_{2}=b\left(1-\chi_{\bar{Q}}\right)$. This induces a splitting $g=g_{1}+g_{2}$ and

$$
\begin{aligned}
m_{Q}\left(g_{1}\right) & =\frac{1}{|Q|} \int_{Q}\left(\int \frac{1}{\varepsilon(y)^{n}} \varphi\left(\frac{x-y}{\varepsilon(y)}\right) b_{1}(y) d y\right) d x \\
& \leqslant \frac{1}{|Q|} \int_{\mathbf{R}^{n}}\left(\int_{\mathbf{R}^{n}} \frac{1}{\varepsilon(y)^{n}} \varphi\left(\frac{x-y}{\varepsilon(y)}\right) d x\right) b_{1}(y) d y \\
& \leqslant \frac{1}{|Q|} \int_{\mathbf{R}^{n}} b_{1}(y) d y \leqslant \frac{1}{|Q|} \cdot k \cdot|\bar{Q}|<c k
\end{aligned}
$$

for a geometric constant $c$. Here we have used the fact that $\int \varphi=1$.

Suppose $x$ and $x^{\prime}$ are in $Q$. Then

$$
\left|g_{2}(x)-g_{2}\left(x^{\prime}\right)\right|=\left|\int_{\mathbf{R}^{n} \backslash \bar{Q}} \frac{1}{\varepsilon(y)^{n}}\left(\varphi\left(\frac{x-y}{\varepsilon(y)}\right)-\varphi\left(\frac{x^{\prime}-y}{\varepsilon(y)}\right)\right) b_{2}(y) d y\right| .
$$

Since $|x-y|$ and $\left|x^{\prime}-y\right|$ are of comparable size on the region of integration, the conditions $|x-y|<\varepsilon(y)$ and $\left|x^{\prime}-y\right|<\varepsilon(y)$ are comparable and thus the integrand is zero unless $\varepsilon(y)>\alpha|x-y|$ for a geometric constant $\alpha$. Let $c$ denote the Lipschitz constant of $\varphi$, and $d$ the diameter of $Q$. Then

\footnotetext{
${ }^{2}$ This variant of Carleson's result follows from reading his proof for $\varphi$ of compact support.
} 


$$
\begin{aligned}
\left|g_{2}(x)-g_{2}\left(x^{\prime}\right)\right| & <c \int_{\mathbf{R}^{n} \backslash \bar{Q}} \frac{1}{\varepsilon(y)^{n}} \frac{\left|x-x^{\prime}\right|}{\varepsilon(y)} b_{2}(y) d y \\
& <c k \alpha^{-n-1} \int_{\mathbf{R}^{n} \backslash \bar{Q}} \frac{d}{|x-y|^{n+1}} d y \\
& <c k \alpha^{-n-1} \int_{|x-y|>d} \frac{d}{|x-y|^{n+1}} d y .
\end{aligned}
$$

Thus

$$
\left|g_{2}(x)-g_{2}\left(x^{\prime}\right)\right|<\beta k
$$

for some geometric constant $\beta$. Now

$$
\begin{aligned}
m_{Q}(g)-\inf _{Q} g & <m_{Q}\left(g_{1}\right)+m_{Q}\left(g_{2}\right)-\inf _{Q} g<m_{Q}\left(g_{1}\right)+m_{Q}\left(g_{2}\right)-\inf _{Q} g_{2} \\
& \leqslant c k+\frac{1}{|Q|} \int_{Q}\left(g_{2}-\inf g_{2}\right)<c k+\beta k,
\end{aligned}
$$

the last estimate by (9). Thus $g$ is in BLO with constant dominated by a multiple of $\|b\|_{\infty}$.

The estimates relating the size of $\alpha, \beta, b$ and $f$ in (2) follow from the estimates in Carleson's theorem, the fact that the BMO norm of a BLO function is dominated by a multiple of the BLO constant, and the observation after the proof of Proposition 2.

IV. Further remarks. 1 . Since BMO is the dual space of $H^{1}$ [3] the theorem just proved can be reformulated as a characterization of $H^{1}$.

COROllary 4. If $f$ is measurable then

$$
\|f\|_{1}+\sup _{\substack{g \in L^{+} \\ g^{*}<\infty \text { a.e. }}}\left|\int f \log g^{*}\right| \simeq\|f\|_{H^{1 .}}
$$

Here $\simeq$ denotes equivalence of norms and the integrals are to be interpreted in a principal value sense. (See [3, p. 632] for a discussion of the appropriate limiting process.)

There is a similarity in appearance between (10) and the result of E. M. Stein [8] which states that for nonnegative functions on the circle

$$
\|f\|_{1}+\left|\int f \log ^{+} f\right| \cong\|f\|_{H^{1}}
$$

Perhaps there is a general result relating $\|f\|_{1},\|f\|_{H^{\prime}}$ and $\left.\left|\int f \log \right| f\right|^{*} \mid$.

2. A function $w$ in $L^{+}$is said to be an $A_{p}$ weight $(1<p<\infty)$ if

$$
\sup _{Q}\left(\frac{1}{|Q|} \int_{Q} w\right)\left(\frac{1}{|Q|} w^{-1 /(p-1)}\right)^{p-1}<\infty
$$

where the supremum is over all cubes. If $w$ satisfies this condition for some $p$ then $\log w$ is in BMO. Hence we can apply our previous results to $\log w$ and then exponentiate. The conclusion is that there are positive constants $\alpha$ and $\beta, A_{1}$ 
weights $w_{1}$ and $w_{2}$ and a function $w_{3}$ which is bounded and bounded away from zero such that $w=w_{1}^{\alpha} w_{2}^{-\beta} w_{3}$. A full analysis of the relationship between $\alpha, \beta, p$ and the function $w$ would be very interesting. This might involve an analysis of BLO functions similar to that performed for BMO functions by Garnett and Jones [4].

3. Y. Meyer [6] has shown that Carleson's theorem is true for spaces of homogenous type (see [3] for definitions and examples) which satisfy certain smoothness conditions. Hence our results are valid in those contexts, with essentially the same proofs. In particular our results are valid for BMO of the circle and of the sphere in $\mathbf{R}^{n}$.

It would be interesting to obtain a proof of our theorem which did not require Carleson's rather difficult result.

4. Many results about functions in BMO have analogs for functions of vanishing mean oscillation (see [3] for definitions). We do now know what the analogs of our results are in that context.

\section{REFERENCES}

1. L. Carleson, Two remarks on $H^{1}$ and BMO, Advances in Math. 22 (1976), 269-277.

2. R. R. Coifman and C. Fefferman, Weighted norm inequalities for maximal functions and singular integrals, Studia Math. 51 (1974), 241-250.

3. R. R. Coifman and G. Weiss, Extensions of Hardy spaces and their use in analysis, Bull. Amer. Math. Soc. 83 (1977), 569-645.

4. J. Garnett and P. Jones, The distance in BMO to $L^{\infty}$, Ann. of Math. (2) 108 (1978), 373-393.

5. F. John and L. Nirenberg, On functions of bounded mean oscillation, Comm. Pure Appl. Math. 14 (1961), 415-426.

6. Y. Meyer, private communication.

7. B. Muckenhoupt, Weighted norm inequalities for the Hardy maximal function, Trans. Amer. Math. Soc. 165 (1972), 207-226.

8. E. M. Stein, Note on the class $L \log L$, Studia Math. 32 (1969), 305-310.

9. E. M. Stein and G. Weiss, Introduction to Fourier analysis on Euclidean spaces, Princeton Univ. Press, Princeton, N. J., 1971.

Department of Mathematics, Washington University, St. Louis, Missouri 63130 